\title{
Two types of speaker's ignorance over the epistemic space in Korean
}

\author{
Arum Kang \& Suwon Yoon*
}

\begin{abstract}
The main goal of this paper is to propose a novel paradigm of the split epistemic ignorance, based on two morphologically related particles in Korean: inka in wh-indefinites vs. $n k a$ in modalized questions. Previous literature assumes the interrogative-indefinite affinity as a reflex of a semantic relationship between interrogative markers and indefinites by introducing a set of propositional alternatives (Alternative Semantics: Kratzer and Shimoyama 2002; Alonso-Ovalle 2006, a.o.). However, we challenge these claims by showing that inka and $n k a$ are distinct lexical items which are distributed in different clause types, hence, a new split for the ignorance system is called for. We propose the semantics under which inka and $n k a$ variants are relativized to the epistemic state of the speaker, M(i) (Giannakidou 1995 et seq.). In particular, we show that: (i) the common denominator of $n k a$ and inka is the fact that they both express the speaker's epistemic indeterminacy; but (ii) the crucial difference arises from a strict dichotomy between the types of alternatives that $n k a$ and inka introduce, i.e., the propositional alternatives for $n k a$ vs. the individual alternatives for $i n k a$.
\end{abstract}

Keywords. wh-indeterminates; modalized questions; anti-specificity; epistemic alternatives

1. Introduction. In Modern Korean, the common denominator of the two morphologically related particles, $n k a$ and inka, is that they both express the speaker's epistemic indeterminacy. The particle inka combines with $w h$-words and conveys the inference that the speaker is unable to identify the precise referent in a given question:

(1) Nwukwu-inka wusungha-ess-ta. \#Nwukwu-key?

who-INKA win-Past-Decl who-Q

'Someone (I don't know who he is) won. Guess who?'

Wh-inka is distinct from other mere existential expressions because it manifests referential deficiency. It never induces epistemically specific interpretations, as evidenced by the "guess who" test (Haspelmath 1997, Aloni and Port 2014), which requires a fixed value. We call whinka an anti-specific indefinite because it is an indicator of the speaker's epistemic uncertainty regarding the identity of the referent without having a fixed value.

On the other hand, $n k a$ attaches at the end of the clause and marks the status of the speaker's uncertain knowledge which maximally weakens her commitment to the truth of a proposition:

a. Con-i wusungca-i-nka?

'Is John possibly the winner?; Maybe John is the winner, or maybe not; John might be the winner.'

\footnotetext{
* We are grateful to the audience at Linguistic Society of America's 90th Annual Meeting for comments and discussion. Part of this paper has been presented at the 23th Japanese and Korean Linguistics Conference at MIT and ICKL \& Harvard-ISOKL 2015 at University of Chicago. All errors are our own. Authors: Arum Kang, University of Chicago (arum@uchicago.edu) \& Suwon Yoon, University of Texas, Arlington (suwon.yoon@uta.edu).
} 


\section{b. \#Ney-ka wusungca-i-nka? you-Nom winner-be-Q \\ 'lit. Are you possibly a winner?'}

$N k a$ forms a non-factual question. The general purpose of an information-seeking question is for the speaker to receive a true answer from the addressee. When using $n k a$, however, the question does not call for any response from the hearer, but only concerns itself with the speaker's lack of knowledge as to the content of the proposition. We term the question in (2) a modalized question and $n k a$ is a marker of a modalized question. A modalized question (MQ) is not a question about factuality, but only about the possibility of the proposition. In this sense, MQs involve modal effects. The infelicity in (2b) confirms the modality: since it concerns the addressee herself, using $n k a$ is infelicitous in any situation where the hearer cannot be assumed to know the answer. In what follows, we show that inka and $n k a$ are distinct lexical items, and each phrase thus occurs in different clause types.

The interrogative-indefinite affinity is found in many natural languages in which an identical morphological paradigm is employed for an interrogative marker and a wh-indefinite: e.g. oo in Malayalam (Jayaseelan 2001, 2008), $k a$ in Japanese (Kuroda 1965; Hagstrom 1998), and wa in Yucatec Maya (Anderbois 2011, 2012). A number of morphosyntactic differences among the interrogative markers and also among the $w h$-indefinite markers suggest that this seemingly interrogative-indefinite affinity is a reflex of the semantic relation between the interrogative markers and the indefinites by introducing a set of propositional alternatives (Alternative Semantics: Kratzer and Shimoyama 2002; Alonso-Ovalle 2006, a.o.). However, we challenge these claims by showing that there is a novel paradigm of epistemic ignorance, which is not captured by means of propositional alternatives. Given this multifunctionality, the crucial question we must ask in connection with the variants between inka and $n k a$ is whether to simply posit two different meanings. If we pursue a unified analysis, however, we must find what is the commonality underlying these apparent variations.

With the full range of phenomena that correlate with the occurrence of $(i) n k a$ in questions/indefinites, we suggest that (i)nka is relativized in terms of the epistemic model, M(i) (Giannakidou 1995 et seq.). Extending the notion of referential vagueness (Giannakidou and Quer 2013; Giannakidou and Yoon 2016) and non-veridical equilibrium (Giannakidou 2013; Giannakidou and Mari 2016), we show how precise such a variation arises by positing distinct types of epistemic alternatives that $n k a$ and inka introduce - propositional alternatives for $n k a$ vs. individual alternatives for wh-inka (Kang 2015). The anti-specific indefinite wh-inka imposes a homogenous epistemic state for the speaker of the minimal variation: there are at least two differing values for the indefinite, and the speaker does not know which value is the actual value. On the other hand, the precondition of the propositional alternatives of $n k a$ needs a presence of heterogeneous modal space. The modal space is partitioned with medium-possibilities of equal size. The speaker is undecided as to where the actual world is; therefore it has a reduced truth commitment.

This paper is organized as follows: in section 2, we explore the properties of the antispecific wh-inka and elaborate on our core proposal, including the referential vagueness condition. In section 3, we propose an analysis for the paradigm of $n k a$ capitalizing on nonveridical equilibrium. In section 4 , we discuss the historical change from $\mathrm{C}$ (wh-questions) to the non-C element (RVIs). This diachronic development leads us to new insights into the formal synchronic analysis, illuminating on the fact that (i)nka is ambiguous between the modalized question marker and the anti-specificity marker. We conclude in section 5 . 
2. Referentially vague indefinite wh-inka. In this section, we explore the semantic properties of wh-inka, as follows: First, a speaker's indeterminacy of the anti-specific indefinite wh-inka comes with the preconditions of the individual alternatives. Second, the precondition of the individual alternatives for wh-inka is only a partial variation as opposed to domain exhaustification which is the core property of the Free Choice item.

\subsection{EMPIRICAL MOTIVATIONS: WH-SOURCE WITHOUT QUESTION MEANING IN WH-INKA. Since} languages with wh-indeterminates have a strong tendency to employ question markers, insights from Hamblin semantics have induced an equivalent analysis in languages employing overt Qmarkers and other particles in a series of works (Kratzer and Shimoyama 2002; Kratzer 2005, a.o.). Kratzer and Shimoyama's analysis is couched in a semantic framework in which the LF of a sentence containing wh-indeterminates is formulated by composing propositional alternatives. Under this analysis, expressions such as a bare wh-indeterminate in $\mathrm{w}$ denote the set of persons in $\mathrm{w}$ and a verb $o$ - 'came-in' denotes the singleton set containing the property of 'come in.' Then the Point-wise Functional Application rule applies to these denotations, as defined below:

(3) Point-wise Functional Application (Kratzer and Shimoyama 2002):

If $\alpha$ is a branching node with daughters $\beta$ and $\gamma$, and $[[\beta]]^{\mathrm{w}, \mathrm{g}} \subseteq \mathrm{D}_{\sigma}$ and $[[\gamma]]^{\mathrm{W}, \mathrm{g}} \subseteq \mathrm{D}_{<_{\sigma, r}>}$, then $[[\alpha]]^{\mathrm{w}, \mathrm{g}}=\left\{\mathrm{a} \in \mathrm{D}_{r}: \exists \mathrm{b} \exists \mathrm{c}\left[\mathrm{b} \in[[\beta]]^{\mathrm{w}, \mathrm{g}} \& \mathrm{c} \in[[\gamma]]^{\mathrm{w}, \mathrm{g}} \& \mathrm{a}=\mathrm{c}(\mathrm{b})\right]\right\}$

Via the Point-wise functional application, the LF of a sentence containing a $w h$-indeterminate is structured by composing propositional alternatives of a higher type, as shown in (4) below.

$$
\begin{aligned}
& {[[\text { who-indeterminate came }]]^{\mathrm{w}, \mathrm{g}}=\left\{\mathrm{p}: \mathrm{x}\left[\operatorname{person}(\mathrm{x})(\mathrm{w}) \& \mathrm{p}=\lambda \mathrm{w}^{\prime} \text {. came }(\mathrm{x})\left(\mathrm{w}^{\prime}\right)\right]\right\}} \\
& =\{\text { Ann came, Bill came, Charles came... }\} \text { (for all men in the world of evaluation) }
\end{aligned}
$$

The propositional alternatives expand until they are closed with a propositional quantifier. Then the semantics of the existential is formulated as below, denoting a singleton set of the proposition:

(5) Where $\mathrm{A}$ is a set of the proposition, we have:

a. $\quad[\exists](\mathrm{A})=$ the proposition that is true in all worlds in which a proposition in $\mathrm{A}$ is true $\}$

b. $\left([[\text { who-indeterminate came }]]^{\mathrm{w}, \mathrm{g}}\right)=\{$ there is at least one person that came in $\mathrm{w}\}$

However, the assumption of the propositional alternatives in the analysis of Korean wh-inka can be refuted on empirical grounds. A closer look reveals that wh-inka never receives a wh-question meaning. The problem, as illustrated below, is that when wh-inka forms a question, it is obligatorily interpreted as a yes-no question. If we follow Hamblin semantics, the existence of the Q-particle $n i$ in (6) cannot account for the lack of interpretation of the wh-question:

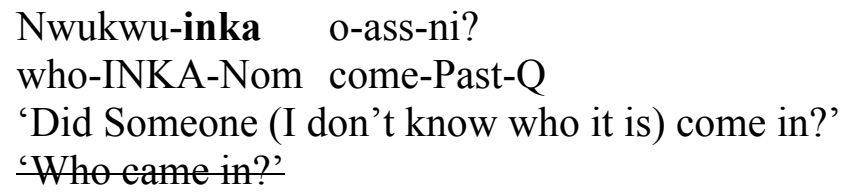

The puzzle is: how do we explain the lack of interpretation of the wh-question here? Unlike English, which employs morphologically distinct lexical items for who and someone, Korean employs the same morphological forms for $w h$-words and the corresponding (in)definites: e.g. $n w u k w u$ 'who/someone'. We consider that inka is only combined with the existential $w h$ indeterminate $n w u k w u$ 'someone'. RVIs are variables that can be bound by existential closure and have a $w h$-indefinite reading, as represented in (7): 
As shown in the denotation in (7), wh-inka is a non-wh-item. The RVI wh-inka and $w h$-item therefore do not have the same source. Given this, Hamblin semantics that rely on the generation of propositional alternatives seems to be an unattractive option.

Here we suggest a different logical schema for wh-indeterminates and proceed with our analysis of $w h$-indeterminates going back to the classical treatment of indefinites as Heimian variables. By treating indeterminates as Kamp/Heim-style indefinites, bare $w h$-words are assumed to have a role similar to that of as yet unbound logical variables (à la Nishigauchi1990; Cheng and Huang 1996, a.o.). Wh-words do not have an inherent quantificational force, but the particles are the binders, which determine the quantificational force of the $w h$-words. The case of classical indefinite interpretation of $w h$-inka can be schematized as follows:

$$
\mathrm{Q}[\mathrm{w}, \mathrm{x}][\ldots \text { indefinite-D NP }(\mathrm{x}, \mathrm{w}) \ldots \mathrm{VP}]
$$

The wh-set undergoes operations on the individual domain in accordance with the individual based variation. Accordingly, by assuming the $w h$-indeterminates are equivalent to the Heimian indefinites, we can easily explain why $w h$-indeterminates lack an interrogative meaning. The determiner is the place where the speaker's distinct epistemic state is reflected, and we assume that the anti-specificity marker inka, as an operator, directly applies to the indeterminate and occupies the D position.

2.2. MARKEDNESS AND ANTI-SPECIFICITY. It is generally agreed upon that the English indefinite article $a(n)$ is unmarked. In fact, it is an unmarked argument marker since it contributes no constraints on the variable it introduces. On the other hand, there are marked indefinite Ds with which the speaker intends to convey something more than just that the referent of the existential indefinite exists. The proliferation of marked indefinite determiners in languages is not redundant, but rather an indication that each determiner is the place where the speaker's epistemic state is differentiated. Whereas (9a) with the indefinite article $a$ merely states that one student came to see me, and (9b) with a certain/a particular implies that a specific student came to see me. The latter has more constrained distribution than the one with the indefinite article $a$. Unlike the unmarked-article-headed indefinite in (9a) which scopes freely, the value of the indefinite in (9b) is fixed and the resulting reading is truth-conditionally equivalent to a reading where the indefinite has a wide scope:

a. A student came to see me.

b. A certain/a particular student came to see me.

Modifiers such as a certain and a particular are called specificity markers. These specificity markers indicate a rigid reference in the speaker's mind. On the other hand, some indefinite determiners convey information about the speaker's lack of knowledge since Haspelmath (1997) includes (lack of) knowledge of the speaker as one of the possible dimensions of variation within the class of indefinites. For example, a wide scope reading is not available for some student in (10) since it does not naturally lend itself to a referential use:

Some student came to see me.

This type of lack of specificity is termed anti-specificity. 
On the nature of (anti-)specific indefinites, Brasoveanu and Farkas (2010) further analyze the non-neutral uses of the specificity markers, suggesting the following categories:

(11) a. Family of specificity markers: Ds that have the semantics of ordinary indefinites + a constraint that leads to relative stability of the reference.

b. Family of anti-specificity markers: Ds that have the semantics of ordinary indefinites + a constraint that leads to relative variability of the reference.

The distinction between specificity and anti-specificity markers depends on the nature of the constraint on D, which contributes different values w.r.t. stability/variability of the reference. With a use of a morphologically overt $\mathrm{D}$ as a specificity marker, in the specific reading, the value of the indefinites is fixed and the speaker's epistemic identifiability is imposed. On the other hand, by choosing to use an anti-specific determiner, the speaker ensures that she has no particular individual in mind. The value of anti-specific indefinites is not fixed and the speaker's epistemic non-identifiability is reflected. An important question arises at this point: what is responsible for the nature of anti-specificity constraints for wh-inka? It is a referentially vague indefinite (RVI) that has been argued to be an indicator of the speaker's epistemic indeterminacy/ignorance about the identity of the referent without having a fixed value.

2.3. NON-IDENTIFIABILITY. In this subsection, we introduce a set of novel data with wh-inka, and the referential vagueness condition. Wh-inka is generally associated with the inference that the speaker is unable to identify the individual in question. The listener is not allowed to ask for a specific value on the reference as seen below:
A: Nwukwu-inka cenhwaha-(e)ss-ta. who-INKA call-Past-Decl
'Someone has called.'
B: \#Nwukwu-i-ess-ni? who-be-Past-Q
'Who was it?'

The speaker's ignorance induced by wh-inka is further supported by the following three identification methods - naming, ostension and description, that Aloni and Port (2014) suggest. As shown below, none of them seem to be compatible with wh-inka:

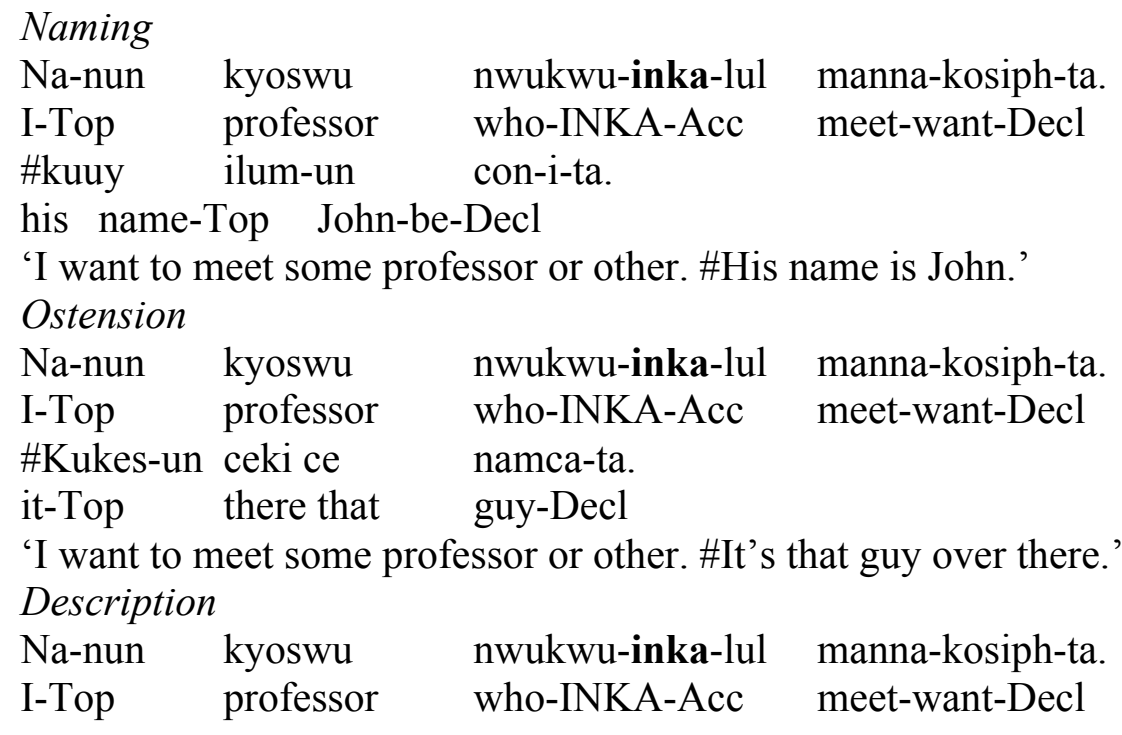


\#ku-nun pwulkun meli-ta.

he-Top red haired-Decl

'I want to meet some professor or other. \#He is red haired.'

The sentences from (13) to (15) exemplify the cases where wh-inka carries the uncertainty implication that the speaker does not have a particular (i.e. identifiable) referent in mind. The second sentences in each set of data introduce a specific value. When the speaker knows what the target value is, the use of the wh-inka becomes odd. This difference exactly parallels with what we find with algún in Spanish, kápjos/kanenas in Greek and wh-rato in Korean (Giannakidou and Quer 2013; Giannakidou and Yoon 2016).

Another set of empirical data supporting the account of referential vagueness comes from the interaction with intentional operators. As shown below, a bare $w h$-item gives rise to the interpretation of either de re or de dicto as in (16) while a reading of wh-inka only yields to de dicto as in (17):
a. Mary-nun uysa nwukwu-hako Mary-Top doctor who/someone-with
b. de re: $\left[\exists \mathrm{x}\right.$ in $\mathrm{w}_{0}$ WANT (marry $\left(\mathrm{x}\right.$ at $\left.\left.\left.\mathrm{w}_{0}\right)\right)\right]$
c. de dicto: [WANT $[\exists \mathrm{w} \exists \mathrm{x} \operatorname{doctor}(\mathrm{x}, \mathrm{w}) \operatorname{marry}(\mathrm{x}$ at $\mathrm{w})]]$ kyelhonha-ko sipehan-ta.
marry-Comp want-Decl
a. Mary-nun uysa nwukwu-inka-hako kyelhonha-ko sipehan-ta. Mary -Top doctor who/someone-INKA-with marry-Comp want-Decl
b. de dicto: [WANT $[\exists \mathrm{w} \exists \mathrm{x} \operatorname{doctor}(\mathrm{x}, \mathrm{w}) \operatorname{marry}(\mathrm{x}$ at $\mathrm{w})]]$

Given the discussion so far, we propose that the RVI wh-inka has the core properties of referential vagueness, as follows:

(18) Properties of the RVI wh-inka:

a. Wh-inka is indefinite;

b. Wh-inka takes a narrow scope with respect to intentional/modal operators;

c. Wh-inka signals the speaker's indeterminacy concerning the identity of the mentioned referent.

The speaker does not have a particular referent in mind, which reflects the epistemic judgment of uncertainty: it requires that the speaker be in a state of uncertainty on the value of the indefinite. The notion of a non-fixed value constraint is subject to anti-specificity, which is sensitive to the knowledge of the speaker.

2.4. NON-EXHAUSTIVE VARIATION. In this subsection, we compare the two types of Korean antispecific indefinites: indefinite RVI wh-inka vs. FCI wh-na. We show how FCIs and RVIs bring about their distinct properties regarding the (non-)exhaustive variation in imperatives and universal modals. The free choice effect is stronger than referential vagueness in the sense that FCIs exhaust all values in the given domain while RVIs do not. Here we compare Korean data with Giannakidou and Quer's (2013) examples with Greek and Spanish RVIs in order to show that the effect is strikingly parallel, which supports our assumption that Korean wh-inka is equivalent to the RVIs in these languages.

First, let's observe deontic universal modals where the FCIs are infelicitous: 
Context: The family is in a dire financial situation and Mary must save face for the family by marrying a rich guy. Lawyers are rich guys, so she needs to marry some lawyer or other, a member of the set 'lawyer'.

(19) a. \#I Maria prepi na pandrefti opjondhipote dhikighoro.

[Greek]

b. \#Maria-nun pyenhosa nwukwu-na-hako kyelhonhay-yaha-n-ta. [Korean] Mary-Top lawyer who-or-with marry-must-Pres-Decl

'lit. Maria must marry any lawyer.'

The presupposition imposes that there will be a value for FCI 'any lawyer' in each $w$ we consider. The result is an undesirably strong statement because Maria cannot marry all the lawyers. In contrast, the use of the RVI in this context is felicitous:

Context: same as above.

(20) a. I Maria prepi na pandrefti kapjon dhikighoro.

b. Maria-nun pyenhosa nwukwu-inka-hako kyelhonhay-yaha-n-ta. [Korean]

Maria-Top lawyer who-INKA-with marry-must-Pres-Decl

'Maria must marry some lawyer or other.'

The sentence becomes plausible in this case since Maria needs to marry some lawyer or other, a member of the set lawyer, without having to run all the values in the set.

Likewise, in epistemic necessity modals, we get a similar contrast:

Context: I am talking with John and I see that he is very informed about Mary's illness.

(21) a. (Tha) prepi na milise me \{kapjon/\#opjondhipote $\}$ giatro. [Greek]

b. Ku-nun uysa nwukwu-\{inka/\#na $\}$-hako iyakiha-nkey [Korean]

he-Top doctor who-INKA-with talk-Rel

pwunmyengha-ta.

must-Decl

'He must have talked with \{some doctor or other/*any doctor\}.'

The FCI $n w u k w u$-na creates an excessively strong statement that forces John to have to talk to every doctor in the hospital; in order to be informed about one's illness you do not have to talk with all doctors.

Second, let's consider invitations and suggestions. FCIs and RVIs influence these contexts in different ways:

Context: A variety of delicious desserts are presented at the buffet in front of Maria. But she does not have much of an appetite. A says:

(22) FCIs:
a. Fae opjodhipote ghiliko!
[Greek]
b. Prueba cualquier dulce!
[Spanish]
c. Kwaca mwues-ina tusey-yo.
[Korean]
cookie what-NA eat-Imp
'Eat all of these cookies!'

(23) RVIs:

a. Fae kanena ghiliko/kanena ap'afta ta ghiliko

[Greek]

b. Prueba algn dulce/alguno de estos dulces!

[Spanish]

c. Kwaca mwues-inka-lul tusey-yo.

[Korean]

cookie what-INKA-Acc eat-Imp 
'Eat some (or other) of these cookies!'

Note the different situations between the FCI vs. RVI-imperatives in (22) and (23). The contrast in interpretations, i.e. exhaustive and non-exhaustive variations induced by FCIs and RVIs, respectively, reveal that FCIs and RVIs show different behaviors with respect to domain exhaustification. With the FCI in (22), we have a strong imperative to eat as many cookies as the addressee wants; we exhaust all available values in the domain of quantification. With the RVI in (23), on the other hand, the imperatives are gentle invitations to eat some sweet or other. In uttering the sentence, the speaker is not inviting the addressee to consider all sweets; she is only inviting the addressee to consider some and try. In this sense, RVIs do not exhaust the domain. What they require is only partial variation in their domain.

2.5. REFERENTIAL VAGUENESS OF $W H-I N K A$. Given the discussion so far, we propose that the referential vagueness of $w h$-inka is defined as follows:

(24) RVI wh-inka:

A sentence of the form $\lambda \mathrm{w} \cdot[[\operatorname{VP}(\alpha)]]^{\mathrm{c}, \mathrm{w}}$ where $\alpha$ is a singular indefinite containing inka, expresses a proposition only on those utterance context $\mathrm{c}$ where the following conditions are fulfilled:

a. Non-identifiability:

$\lambda w .[[\operatorname{VP}(\alpha)]]^{c, w}$ is defined for a given context $c$, iff the speaker $\mathrm{s}$ of $\mathrm{c}$ is unable to identify a referentially vague variable of $\alpha$

b. Referential vagueness

$\exists \mathrm{w} 1, \mathrm{w} 2 \in \mathrm{W}:[[\alpha]]^{\mathrm{w} 1} \neq[[\alpha]]^{\mathrm{w} 2}$;

[[wh-inka came $]]$ will be defined in c, only if: $\exists \mathrm{w} 1, \mathrm{w} 2 \in \mathrm{W}:[[\alpha]]^{\mathrm{w} 1} \neq[[\alpha]]^{\mathrm{w} 2}$; where $\alpha$ is the referentially vague variable;

if defined, [[wh-inka came]] is true if there is at least one assignment $\mathrm{g}$ that verifies the condition someone $(\mathrm{x}) \&$ came $(\mathrm{x})$.

By using RVI wh-inka, the speaker intends to refer to a vague individual in the world of utterance. It is available because the speaker believes there is more than one value and the $w h$ inka receives distinct values in the contextual domain. This definition imposes a weaker demand on the context in which there are simply at least two alternative worlds where the RVI wh-inka receives distinct values. This is equivalent to the sentences with a plain indefinite - but we still need to capture the referential vagueness effect of the existential-like quantification. This effect is a presupposition of a non-exhaustive variation that the RVI, but not the regular indefinite, gives rise to. These individual alternatives are determined by the denotation of the wh-words within the set of worlds compatible with the speaker's belief in the world of evaluation, which is equivalent to the speaker's epistemic state. Building on Giannakidou (1999, 2009), we model an individual's epistemic state as a set of doxastic alternatives, called a belief model, i.e. a function that gives a set of possible worlds compatible with what $x$, the bearer of the attitude, believes in the actual world:

(25) Belief model of an individual (Giannakidou 1999: (45))

Let $\mathrm{c}=<\mathrm{cg}(\mathrm{c}), \mathrm{W}(\mathrm{c}), \mathrm{M}, \mathrm{s}, \mathrm{h}, \mathrm{w} 0, \mathrm{f}, \ldots>$ be a context.

A model $M_{B}(x) M$ is a set of world associations with an individual $x$ representing world compatible with what $\mathrm{x}$ believes. 
(26) a. John won the race.

b. $\quad[$ John won the race $]=1$ iff

$\forall \mathrm{w}\left[\mathrm{w} \in \mathrm{M}_{\mathrm{B}}(\mathrm{s}) \rightarrow \mathrm{w} \in \lambda \mathrm{w}^{\prime}\right.$. John wins the race in $\left.\mathrm{w}^{\prime}\right]$

If the RVI nwukwu-inka 'someone (I don't know who it is)' is assigned a non-fixed value, i.e. in the narrow scope with respect to the attitude, we may have different visitors in each world:

(27) No particular individual in mind = no fixed value in $\mathrm{M}_{\mathrm{B}}(\mathrm{s})$ :

w1 $\rightarrow$ John, w2 $\rightarrow$ Bill, w3 $\rightarrow$ Charles $\ldots$

The worlds w1, w2 are epistemic alternatives of the speaker: w1,w2 M(speaker), where $\mathrm{M}$ (speaker) is the speaker's belief state, the set of worlds compatible with what he or she believes/knows.

Via the individual anchor, the belief worlds are available as a parameter of evaluation. Variation is modeled as different values in at least two worlds.

3. Modalized question marker $\boldsymbol{n k a}$. In section 3, we show that $n k a$ is a special subtype of the epistemic subjunctive, which indicates an epistemic possibility. The semantic contribution of $n k a$ presupposes the presence of heterogeneous epistemic space. We offer a precise way of capturing the epistemic possibility by nonveridicality_but also showing that the nonveridicality needed is of the weakest form, corresponding to non-veridical equilibrium (Giannakidou 2013; Giannakidou and Mari 2016).

3.1. NKA IN SUBORDICATE CLAUSES. In this subsection, we discuss the behavior of $n k a$ in certain embedded contexts. Given the set of empirical data, we argue that $n k a$ is sensitive to nonveridicality, in the sense that it does not entail the truth of the propositions in subordinate clauses. First, the addition of $n k a$ produces a weakening, nonveridicality effect. As shown below, the $n k a$ collocating with po 'see' gives rise to the meaning of 'it seems that:'

$\begin{array}{llcl}\text { Con-i } & \text { (ama) } & \text { wusungca-i-nka } & \text { po-ta. } \\ \text { John-Nom } & \text { maybe } & \text { winner-be-NKA } & \text { see-Decl } \\ \text { 'lit } & \text { It seems that John }\end{array}$

'lit. It seems that John is the winner.'

Furthermore, when $n k a$ combines with a morphologically negative verb like $m o l$ 'not know,' it gives rise to a dubitative reading:

$\begin{array}{llll}\text { B-ka } & \text { mac-nun } & \text { tap-i-nka } & \text { mol-la. } \\ \text { B-Nom } & \text { correct-Rel } & \text { answer-be-NKA } & \text { not.know-Decl } \\ \text { (ama) } & \text { tap-un } & \text { A-ilkes-i-ta. } & \\ \text { maybe } & \text { answer-Top } & \text { A-might-be-Decl } & \end{array}$

'I doubt if it is a correct answer. The answer is probably A.'

Such a dubitative reading typically involves the subjunctive mood since it is related to the speaker's commitment with regard to irrelis. It is further supported by the fact that $n k a$ sentences are compatible with probability adverbs like ama(to) 'probably' above, but incompatible with necessity adverbs such as pwunmyenghi 'certainly:'

$\begin{array}{lll}\text { B-ka } & \text { mac-nun } & \text { tap-i-nka mol-la. } \\ \text { This } & \text { correct-Rel } & \text { answer-be-NKA not know-Decl } \\ \text { \#pwunmyenghi } & \text { tap-un } & \text { A-ilkes-i-ta. } \\ \text { certainly } & \text { answer-Top } & \text { A-might-be-Decl }\end{array}$

'I doubt if it is the correct answer. The answer will certainly be A.' 
The nonveridical nature of the Korean MQ is further shown by considering what kind of predicates normally combine with it. Indicative predicates such as assertive, epistemic, factive, and semi-factive verbs are incompatible with MQ:

(31) Verbs that do not combine with the MQs: indicative in Korean (Yoon 2013:(48a-d))

a. assertive: malha 'say', ilk 'read', cwucangha 'claim'

b. epistemics: mit 'believe'

c. factive verbs: kippu 'glad', al 'know', hwuhyoha 'regret'

d. semi-factives: kkaytat 'discover', kiekha 'remember'

Rather, verbs that combine with $n k a$ are characterized as verbs of uncertainty, i.e. ones that do not combine with nonveridicals (Yoon 2011, 2013):

(32) Verbs that do not combine with MQs: subjunctives in Korean (Yoon 2013:(49a-d))

a. volitional: hyimangha 'hope', kitayha 'hope'

b. verbs of fear: twuryeweha 'fear', kekcengha 'worry'

c. directives: chwungkoha 'advise', ceyanha 'suggest'

d. verbs of uncertainty: moru 'not;know', kwungkumha 'wonder'

Again, it supports our claim that $n k a$ does not entail the truth of the proposition in a subordinate clause and reduces the speaker's commitment to the truth of the sentence. In what follows, we show that the uncertainty may originate from the subject's presumption of the non-high possibility of the proposition given by contextually available information.

3.2. MEDIUM POSSIBILITY OF MQ. Thus far, we have observed that $n k a$ in MQs implicates that a subject is uncertain as to whether the propositional content is possible or not. It is further evidenced by the fact that an MQ with $n k a$ is infelicitous in contexts with high possibility and low possibility, while felicitous in contexts with medium possibility:

imsin-i-nka?

pregnancy-be-NKA

'Is it possibly a pregnancy?'

(34) MQ and Degree of Certainty

a. High-possibility context (80-100\%): It has been 1 year since my sister got married. One day, I visited her. She wanted to tell me about the surprise news. She showed me her pregnancy test. There were two lines on it. I say: continuation (33): \#

b. Medium-possibility context (50\%): It has been 1 year since my sister got married. One day, I visited her. She showed some symptoms that she was suffering from morning sickness, and craved eating something sour. I say: continuation by (33): o.k.

c. Low-possibility context (0-20\%): High-likelihood context (80-100\%): It has been 1 year since my sister got married. One day, I visited her. She told me that she wants a baby. I say: continuation by (33): \#

The example in (34) suggests that in order for $n k a$ to be felicitous, the speaker must believe that the realization of the propositional content has a medium possibility. The context is set up such that the speaker is uncertain about the truth of the proposition expressed by the sentence: the speaker does not know which of $\mathrm{p}$ (she is pregnant) and non- $p$ (she is not pregnant) is true. However, if the evidence points too strongly, as in (34a), or too weakly, as in (34c), in favor of the proposition being true, $n k a$ becomes infelicitous. The contribution of $n k a$ thus involves medium certainty. By using MQ, a speaker presupposes the medium possibility of realization of 
the propositional content. We can further argue that having $50 \%$ certainty can naturally be explained if the core reanalysis of $n k a$ necessarily occurs in a statement whose meaning consists of both $p$ and non- $p$. The speaker's presupposition on the medium possibility of the realization of the propositional content, we argue, is the reason why $n k a$ is used.

3.3. NON-VERIDICAL EQUILIBRIUM OF MQ. Our proposal is that the semantic contribution of $n k a$ is to function as a nonveridical commitment-weakening operator and gives rise to the speaker's weakest commitment. We suggest that the precondition of $n k a$ comes with two main parts: (i) the epistemic constraints of the MQ marker can be achieved by means of the presence of the nonveridical modal space; and (ii) this modal space is partitioned in the equipoised epistemic space.

Nonveridicality is placed at the heart of modality mood (Giannakidou 1995 et seq.).

Giannakidou assumes Kratzerian semantics for all modals, where modals take modal bases and ordering sources, and add one ingredient, the Nonveridicality Axiom, which all modal bases are nonveridical. ${ }^{1}$ As shown below, nonveridicality is a precondition on modalities, where nonveridical domains are sets of worlds partitioned into $p$ and non-p worlds:

(35) The Nonveridicality Axiom of modals (Giannakidou and Mari 2016: (27)):

$\operatorname{MODAL}(\mathrm{M})(p)$ can be defined only if the modal base $\mathrm{M}$ is nonveridical, i.e. only if $\mathrm{M}$ contains $p$ and non- $p$ worlds.

This axiom guarantees that MODAL $p$ will not entail $p$, since there are $p$ worlds in $\mathrm{M}$, and the actual world may be one of those. The modal base $\mathrm{M}$ intersects with $p$, but also contains non- $p$ worlds. The modal is biased when it delivers a positive bias: if the set of $p$ worlds is larger than the set of non- $p$ worlds. Existential modals do not have bias and their modal base is partitioned into $p$ and non- $p$ sets of equal size (i.e. nonveridical equilibrium; Giannakidou 2013). The

1 Here we introduce the fundamental notions of the nonveridical modal. First, ordering sources and Best worlds are defined in the sense of Portner (2009) as follows:

(1) Ordering of worlds (Portner 2009, p.65):

For any set of propositions $\mathrm{X}$ and any worlds $\mathrm{w}, \mathrm{v}$ : $\mathrm{w}<\mathrm{X} v$ iff

(i) for all $\mathrm{p} \in \mathrm{X}$, if $\mathrm{v} \in \mathrm{p}$, then $\mathrm{w} \in \mathrm{p}$; and

(ii) for some $\mathrm{q} \in \mathrm{X}, \mathrm{w} \in \mathrm{q}$ and $\mathrm{v} \notin \mathrm{q}$

Second, given an epistemic modal base M(i), the best option is defined as a function over M(i):

(2) For any set of propositions X, the best option for worlds is a function over M(i) (Giannakidou \& Mari 2016: (30)):

$$
\operatorname{BestSM}(\mathrm{i})=\left\{\mathrm{w}^{\prime} \in \mathrm{M}(\mathrm{i}): \forall \mathrm{q} \in \mathrm{X}\left(\mathrm{w}^{\prime} \in \mathrm{q}\right)\right\}
$$

Third, the best worlds consist of two basic parts: support and bias. The support function takes the modal base as its argument and returns a subset of it. The set of worlds returned is such that the propositions in the ordering source $\mathrm{S}$ are true.

(3) Support function (Giannakidou \& Mari 2016: (31)):

$\operatorname{SupportSM}(\mathrm{i})=\mathrm{X}$ s.t. $\mathrm{X} \subset \mathrm{M}(\mathrm{i}) \& \forall \mathrm{w}^{\prime} \in \mathrm{X}: \mathrm{p}\left(\mathrm{w}^{\prime}\right)$

The support set is the inner domain of the modal base, and the modal base is its outer domain. The support function delivers the positive set of the nonveridical modal base. Bias is defined in terms of a measure function $\mu$, which takes sets as arguments and returns their sizes.

(4) A modal is biased iff (Giannakidou \& Mari 2016: (32)):

$\mu(\operatorname{SupportS}(\mathrm{M}(\mathrm{i}))>\mu \mathrm{M}(\mathrm{i})) \backslash(\operatorname{SupportS}(\mathrm{M}(\mathrm{i})))$

The modal is biased when it delivers positive bias: if the set of $\mathrm{p}$ worlds is larger than the set of $\neg \mathrm{p}$ worlds. 
nonveridical equilibrium is a state of fifty-fifty and $p$ and non- $p$ are equal options. The nonveridical equilibrium can be generalized as follows:

(36) Nonveridical equilibrium (general) (Giannakidou and Mari 2016: (34))

Let $\mathrm{X}$ s.t. $\mathrm{X} \subset \mathrm{M}(i)$.

A modal base $\mathrm{M}(i)$ is a non-veridical equilibrium iff $\mu(\mathrm{X}) \approx \mu(\mathrm{M}(\mathrm{i}) \backslash \mathrm{X})$

The nonveridical modal base of $n k a-p$ holds the non-veridical modal space, partitioned in equipoise that are compatible with the speaker's belief, and indicates an equal possibility of its spaces given what the speaker's doxastic (or belief) world is: i.e. $n k a$ conveys that the speaker considers both $p$ sets and non- $p$ sets equally possible.

Epistemic modality specifies the degree of a speaker's certainty about a proposition in questions. The commonality between $n k a$ and the epistemic modal might is that they both express that a proposition is held in at least one of the speaker's doxastic alternatives. The speaker must believe that the proposition is a possibility given what she knows to be true. We thus suggest that an appropriate interpretation of $n k a$ is obtained by considering the epistemic status of the speaker. It is characterized as equipoised epistemic space.

(37) Non-veridical equilibrium of a modalized question

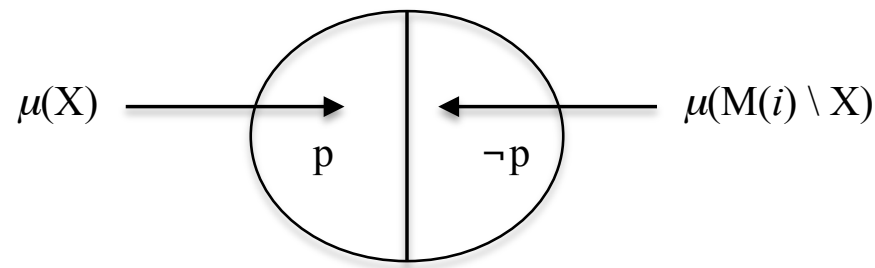

(38) $[[\mathrm{NKA}(p)]]^{M, i}$ will be defined if only if

(i) the modal base $\mathrm{M}(i)$ is nonveridical;

(ii) $\exists \mathrm{X} \subset \mathrm{M}(i)$ s.t. $\mu(\mathrm{X}) \approx \mu(\mathrm{M}(i) \backslash \mathrm{X})$ (nonveridical equilibrium)

if defined, $[[\mathrm{NKA}(p)]]^{\mathrm{M}, \mathrm{i}}=1$ iff $\forall w^{\prime} \in \mathrm{X}$ s.t. $\mathrm{X} \subset \mathrm{M}(i)$

The speaker has reduced the truth commitment by creating a nonveridical modal space: i.e. one that contains $p$ and non- $p$ worlds. The domains of non-veridical equilibrium are modal domains partitioned into $p$ and non- $p$ worlds. Unlike the typical partition, which is the result of an ordering (e.g. ordering sources with modals), we show that there is no best world in $n k a$ questions hence no ordering occurs.

4. Path to grammaticalization of referentially vague indefinites: from question to indefinite. It has been argued that inka went through grammaticalization and was reanalyzed as a single nominal particle (i.e. determiner) from the MQ marker $n k a$ (Yoon 2005). In this subsection, we investigate the sources of the RVI wh-inka, and examine their development from Old Korean into Modern Korean, including their spread into new environments and the development of its ignorance component. We show that wh-inka has historically undergone a reanalysis from $\mathrm{C}$ (i.e. wh-questions: $w h-i-n k a$ ' $w h$-be-Q') to non-C elements (i.e. referentially vague indefinites: $w h$ inka ' $w h$-INKA'). An understanding of these diachronic developments leads us to new insights into the formal synchronic analysis.

Until the end of the 19th century, wh-phrases such as $n w u k w u$ 'who' in Korean were used exclusively as interrogative pronouns and only in questions. What the data in Modern Korean suggest is that the distribution of $n w u k w u$, for some reason, has been extended to non- 
interrogative contexts. This means that the properties of $w h$-words in Korean have changed over time from the exclusively interrogative pronouns to quantificational indefinite pronouns. The distribution of $w h$-phrases in Modern Korean has been extended to non-interrogative contexts (C-M Suh 1987, Kim 1992, a.o.). To explain the extension, it can be assumed that Q-morphemes lost their syntactic status as interrogative $\mathrm{C}$ and underwent reanalysis to non-C elements with varying quantificational forces such as an existential quantifier (Kim 1992). This change, in turn, would have had the consequence of allowing $w h$-phrases to appear in genuinely noninterrogative contexts. According to this hypothesis, the sequence of changes must have proceeded in the following order (Yoon 2005: (43)):

(38) a. wh-QPs like nwukwu-inka lost their clausal identities.

b. inka was reanalyzed as the existential quantifier.

c. $\quad w h$-words like $n w u k w u$ were reanalyzed as not exclusively interrogative.

d. $\quad w h$-words like $n w u k w u$ came to be used as a bare indefinite pronoun.

The idea is that although Q-morphemes historically were all Cs, some of them lost their syntactic status as $\mathrm{C}$ and were reanalyzed as non-C elements with varying quantificational forces. Now they can be used in both interrogative and non-interrogative contexts: when they are associated with an interrogative $\mathrm{C}$, they are interpreted as interrogative pronouns, while when they are associated with an existential quantificational inka, they read as an existential pronoun.

With this background, we can further argue that the change of $w h$-words from interrogative to indefinite pronouns with quantificational variability can be naturally explained if the core reanalysis of wh-QPs like nwukwu-inka is from an interrogative $\mathrm{C}$ to an RVI-marker. Wh-inka, which had an interrogative $\mathrm{C}$ before the reanalysis, now has an existential quantificational force as an RVI. The reanalysis of wh-inka that triggered such a change, we propose, is as follows:

\begin{tabular}{lll} 
wh-questions $>$ RVIs & \\
Stage & Conceptual schema & Function \\
\hline Stage 1 & $\begin{array}{l}\text { X encodes a } \\
\text { equipoised partition on } \\
\text { the proposition }\end{array}$ & MQ-marker \\
Stage 2 & $\begin{array}{l}\text { X encodes the } \\
\text { speaker's } \\
\text { epistemic uncertainty } \\
\text { on the reference }\end{array}$ & Anti-specificity marker \\
\hline
\end{tabular}

Table 1: Semantic change of (i)nka

In the historical reanalysis process, the original properties of a construction before the reanalysis may continue to constrain the use of the reanalyzed forms (Hopper and Traugott 2003). This can account for why the indefinite wh-inka conveys the speaker's ignorance: the speaker's ignorance induced by the RVI wh-inka came from the MQ, which is based on the original properties of a construction prior to the reanalysis. Therefore, the reanalyzed RVIs headed by the particle inka, which was originally a MQ, are unnatural in a situation where the speaker knows the identity of the referent.

5. Conclusions. In this paper, we suggest a novel paradigm of the split epistemic ignorance, based on two particles in Korean: the anti-specificity marker inka in wh-indefinites vs. the epistemic subjunctive marker $n k a$ in modalized questions. We showed that inka and $n k a$ are 
distinct lexical items, introducing individual and propositional alternatives, respectively. A theoretical implication of the current study is then that the Korean facts bring a fresh perspective with regard to the notion of the speaker's uncertainty over the epistemic space. We suggest the necessity of a strict dichotomy of epistemic ignorance which gives rise to the differing semantics of referential vagueness and nonveridical equilibrium.

\section{References}

Alonso-Ovalle, Luis. 2006. Disjunction in alternative semantics. University of Massachusetts Amherst dissertation.

Aloni, Maria, and Angelika Port. 2014. Epistemic indefinites and methods of identification. In Luis Alonso Ovalle and Paula Menendez-Benito (eds.), Epistemic Indefinites. Oxford University Press.

AnderBois, Scott. 2011. Issues and alternatives. University of California Santa Cruz dissertation.

AnderBois, Scott. 2012. Focus and uninformativity in Yucatec Maya questions. Natural Language Semantics 20.349-390. http://dx.doi.org/10.1007/s11050-012-9084-3.

Cheng, Lisa L.-S. and James C.-T Huang. 1996. Two types of donkey sentences. Natural Language Semantics 4.2: 121-163.

Giannakidou, Anastasia. 1995. Subjunctive, habituality and negative polarity items. Semantics and Linguistic Theory 5, 94-111.

Giannakidou, Anastasia. 1999. Affective dependencies. Linguistics and Philosophy 22: 367-421.

Giannakidou, Anastasia. 2009. The dependency of the subjunctive revisited: temporal semantics and polarity. Lingua 120.1883-1908. http://dx.doi.org/10.1016/j.lingua.2008.11.007.

Giannakidou, Anastasia. 2013. Inquisitive assertions and nonveridicality. In The dynamic, inquisitive, and cisionary life of $\phi, ? \phi$ and possibly $\phi$, A festschrift for Jeroen Groenendijk, Martin Stokhof and Frank Veltman, ed. by Maria Aloni, Michael Franke, F. Roelofsen: 115126.

Giannakidou, Anastasia and Josep Quer. 2013. Exhaustive and non-exhaustive variation with free choice and referential vagueness: Evidence from Greek, Catalan, and Spanish. Lingua, (126): 120-149. http://dx.doi.org/10.1016/j.lingua.2012.12.005.

Giannakidou, Anastasia and Suwon Yoon. 2016. Scalar marking without scalar meaning: nonscalar, non-emphatic EVEN-marked NPIs in Greek and Korean. Language. (To appear)

Giannakidou, Anastasia and Alda Mari. 2016. The semantic roots of positive polarity: epistemic modal verbs and adverbs. Ms., University of Chicago.

Hagstrom, Paul A. 1998. Decomposing questions. Massachusetts Institute of Technology dissertation.

Haspelmath, Martin. 1997. Indefinite pronouns. Oxford University Press.

Hopper, Paul and Elizabeth Traugott. 2003. Grammaticalization (2nd edition). Cambridge: Cambridge University Press.

Jayaseelan, K. A. 2001. Questions and question-word incorporating quantifiers in Malayalam. Syntax 4, 63-93. http://dx.doi.org/10.1111/1467-9612.00037.

Jayaseelan, K. A. 2008. Question particles and disjunction. Ms., The English and Foreign Languages University.

Kang, Arum. 2015. (In)definiteness, disjunction and anti-specificity in Korean: A study in the semantics-pragmatics interface. University of Chicago dissertation. 
Kratzer, Angelika. 2005. Indefinites and the operators they depend on: From Japanese to Salish. In: Carlson, G.N., Pelletier, F.J. (Eds.), Reference and Quantification: The Partee Effect. CSLI Publications, Stanford, 113--142.

Kratzer, Angelika and Junko Shimoyama. 2002. Indeterminate pronouns: The view from Japanese. In 3rd Tokyo conference on psycholinguistics.

Kuroda, Sige-Yuki. 1965. Generative grammatical studies in the Japanese language. Massachusetts Institute of Technology dissertation.

Kim, C.-H., 1992. A study on interrogative and indefinite pronouns in Korean (written in Korean). Hanyang University dissertation.

Nishigauchi, Taisuke. 1990. Quantification in the theory of grammar. Dordrecht: Kluwer Academic Publishers.

Suh, C.-M. 1987. Study of Questions in Korean (in Korean). Seoul: Tap Publishing, Co.

Yoon, Jeong-Me. 2005. Two historical changes in wh-constructions in Korean and their implications. Studies in Generative Grammar, 15: 457-487.

Yoon, Suwon. 2011. 'Not' in the mood: The syntax, semantics, and pragmatics of evaluative negation. University of Chicago dissertation.

Yoon, Suwon. 2013. Parametric variation in subordinate evaluative negation: Korean/Japanese versus others. Journal of East Asian Linguistics, 22: 133-166.

http://dx.doi.org/10.1007/s10831-012-9100-0. 